\title{
Percepciones sociales de la Selección Chilena de Fútbol como marca
}

Social perceptions of the Chilean National Soccer Team as a brand

Percepções sociais da Seleção Chilena de Futebol como marca
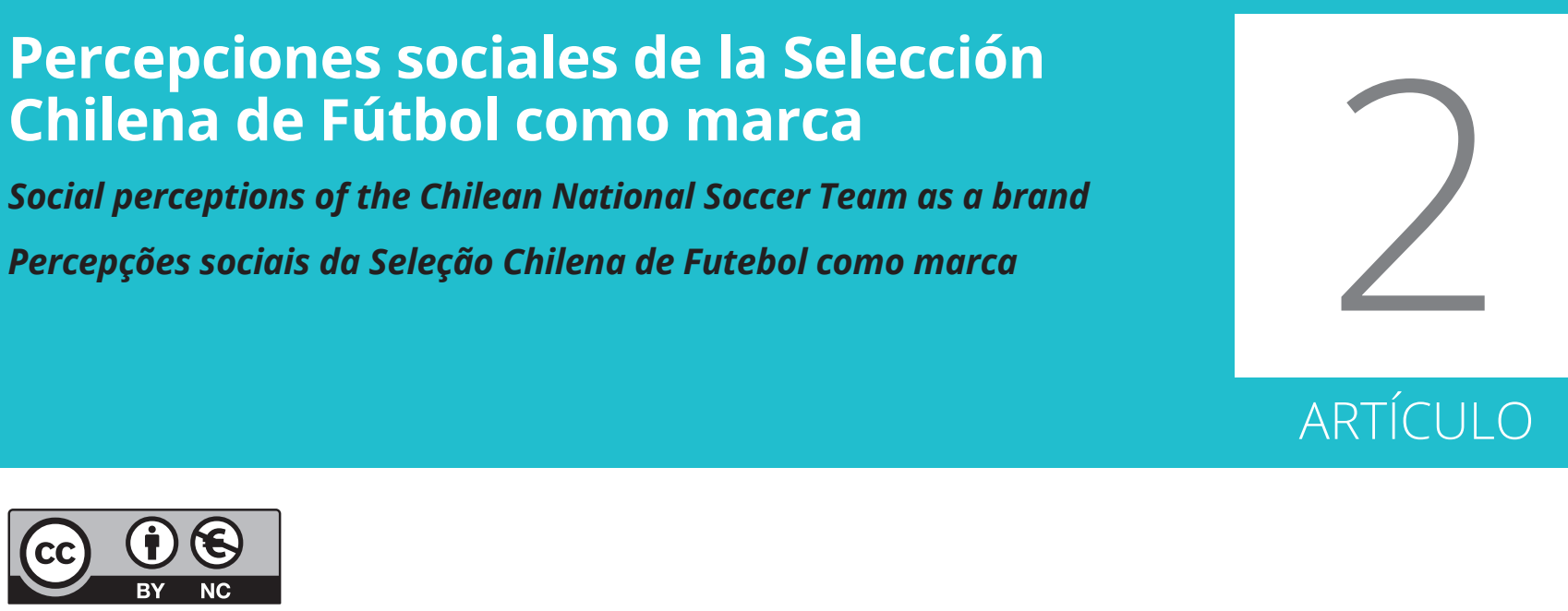

\section{Cristóbal Benavides}

Universidad de los Andes (Chile)

Decano y profesor de la Facultad de Comunicación de la Universidad de los Andes, Chile. Doctor en Comunicación Pública por la Universidad de Navarra, España.

cbenavides@uandes.cl

orcid.org/ 0000-0001-5573-6785

\section{Juan Ignacio Brito}

Universidad de los Andes (Chile)

Profesor de la Universidad de los Andes, Chile. Master of Arts in Law and Diplomacy por Fletcher School of Law and Diplomacy, Tufts University, Boston, Estados Unidos.

jbrito@uandes.cl

orcid.org/ 0000-0003-0147-8690

\section{Juan Ignacio Guerrero}

Universidad de los Andes (Chile)

Profesor de la Facultad de Comunicación de la Universidad de los Andes, Chile. Magíster en Comportamiento del Consumidor, Universidad Adolfo Ibáñez, Chile.

JJGUERRERO@uandes.cl

orcid.org/ 0000-0002-7286-3660 


\section{Francisco Tagle}

Universidad de los Andes (Chile)

Profesor de la Facultad de Comunicación de la Universidad de los Andes, Chile. Doctor en Estudios Latinoamericanos, Universidad de Salamanca, Chile.

fjtagle@uandes.cl

orcid.org/0000-0003-1480-1020

RECIBIDO: 10 de agosto de 2020 / ACEPTADO: 02 de diciembre de 2020

\section{Resumen}

Los éxitos deportivos alcanzados por la Selección Nacional de Fútbol de Chile en los años 2015 y 2016 permiten distinguir los cambios en las percepciones de los chilenos respecto a La Roja. A partir de la consideración de este equipo como una marca, se concluyó que posee un alto grado simbólico en cuanto a elemento de unión de la sociedad chilena, a la que se considera como fragmentada. Para este estudio se realizaron 2.410 encuestas cara a cara en Santiago de Chile a personas de entre 10 y 80 años, que fueron segmentadas a partir de los grupos socioeconómicos que conforman el país.

\section{PALABRAS CLAVE:}

Chile, Selección Nacional de Fútbol, Marca, Percepciones, Aaker.

\section{Abstract}

The sporting successes achieved by the Chilean National Soccer Team in 2015 and 2016 allow us to distinguish the changes in the perceptions of Chileans regarding La Roja. Based on the consideration of this team as a brand, it was concluded that it has a high symbolic degree in terms of a unifying element of Chilean society, which is considered fragmented. For this study, 2,410 face to face surveys were conducted in
Santiago de Chile with people between 10 and 80 years of age, which were segmented based on the socioeconomic groups of the country.

\section{KEYWORDS}

Chile, National Soccer Team, Brand, Perceptions, Aaker.

\section{Resumo}

Os sucessos esportivos alcançados pela Seleção Chilena de Futebol em 2015 e 2016 permitem distinguir as mudanças nas percepções dos chilenos em relação a La Roja. A partir da consideração desta equipe como marca, concluiu-se que ela possui um alto grau simbólico em termos de um elemento unificador da sociedade chilena, que é considerada fragmentada. Para este estudo, foram realizadas 2.410 pesquisas presenciais em Santiago do Chile com pessoas entre 10 e 80 anos, as quais foram segmentadas com base nos grupos socioeconômicos que compõem o país.

\section{PALAVRAS-CHAVE}

Chile, Seleção Nacional de Futebol, Marca, Percepções, Aaker. 


\section{INTRODUCCIÓN}

La clasificación de Chile al mundial de fútbol de Brasil 2014 marcó un hito en el deporte nacional. Por primera vez en la historia se lograba asistir de manera consecutiva a un mundial sin contar con la ventaja de la localía. Se rompía, de esa manera, una barrera infranqueable para el equipo chileno. Este hecho histórico no solo abarcó una dimensión deportiva, sino que también captó un alto grado de atención y en la sociedad chilena. Así el grado de engagement, relación construida en base a una serie de atributos y experiencias positivas que permiten desarrollar fuertes lazos de compromiso (Belair-Gagnon et al., 2019) y lealtad hacia un producto o servicios (Brodie et al., 2013), la transformaron en una de las marcas más reconocidas del país (Tapia, 2019).

Según las estadísticas de la Confederación Sudamericana de Fútbol'1, La Roja (como se le Ilama localmente a la selección nacional), fue la escuadra que más público llevó a los estadios durante las últimas clasificatorias, con 351.440 espectadores. Un promedio de 43.930 personas por partido que dejó atrás en el ranking a los combinados de Colombia, Uruguay y Argentina. Este fenómeno social, que ha despertado la selección chilena en el país, ha sido estudiado desde diferentes perspectivas y dimensiones. Algunos autores lo hicieron desde el nacionalismo (Santa Cruz, 2010), otros desde la política (Hite, 2004; Matamala, 2001), otros desde el marketing (Feller Villanueva, 2006), e inclusive desde el punto de vista económico (Sertsios, 2007).

La presente investigación estudia las diferentes percepciones ciudadanas respecto a los éxitos deportivos alcanzados por la selección nacio-

1 https://www.foxsports.com.mx/news/125153-la-roja-laque-llevo-mas-publico nal entre 2015 y 2016, el periodo más exitoso de su historia, dado que estos ofrecen la posibilidad de distinguir y reconocer diferentes impresiones tanto etarias como socioeconómicas en cuanto a la personalidad de su marca. Asimismo, en una sociedad chilena que, a pesar de los notables avances de las últimas décadas en reducción de la pobreza, es considerada desigual tanto social como económicamente (PNUD, 2017) y con un profundo malestar hacia las élites (CEP, 2016; Peña, 2020), resulta interesante también el estudio de esta exitosa Selección como símbolo de cohesión entre los diferentes grupos socioeconómicos.

Para eso se encuestaron cara a cara a 2.410 personas pertenecientes a los distintos segmentos etarios y socioeconómicos del país. Todo esto, pocos meses después de que La Roja obtuviera el campeonato de la Copa América Centenario realizada en Estados Unidos, torneo que se llevó a cabo para celebrar los 100 años de esta competencia y en el que participaron las mejores selecciones del continente.

\section{LA ROJA COMO MARCA}

Como señala Anderson (1993), las naciones son comunidades imaginarias unidas por lazos que entregan sentido de pertenencia e identidad a sus ciudadanos y los hacen sentir parte de un todo. En América Latina, como comprueban Santos (2003) y Alabarces (2003), uno de los generadores de dichos lazos son las selecciones nacionales de fútbol.

El fútbol, el estadio, el propio juego y todo lo que sucede alrededor antes, durante y después del momento de competición cobran por lo general un sentido ritual y, según algunos, casi religioso (Acuña \& Acuña, 2016). Como señala Villena (2003, p.26), el fútbol "actúa de 
arena pública", en el proceso de construcción de identidades sociales y culturales de distintos orígenes y niveles.

Por esta razón es que se abre un abanico de dimensiones por la que puede ser estudiada una selección nacional de un país, siendo una de ellas el comprenderla como una marca.

La personalidad de la marca juega un rol fundamental a la hora de realizar una elección (Hernández et al. 2018) y puede ser vital en el éxito o fracaso de un producto, un servicio o alguna actividad de entretenimiento, ya que permite que los consumidores se conecten y construyan relaciones idealmente duraderas y difíciles de romper (Doyle, 1990; Rather et al., 2018).

Uno de los primeros investigadores en estudiar la importancia de la marca desde el punto de vista sicológico fue Allport (1961), quien hizo un paralelo entre una marca y una persona. Así las características de la marca y los comportamientos de esta podrían ser fácilmente reconocibles por los consumidores. La relación entre ambos dependería de la identificación que lograran establecer a lo largo del tiempo. Aaker y Biel (1993), en la misma línea, afirman que una organización puede tener y mantener buenas relaciones con sus clientes si logra trabajar bien su personalidad con el objetivo de ir mejorando su valoración y reconocimiento en el mercado. Blackket (2009) da un paso más allá al sostener que las marcas más potentes son aquellas construidas desde la base de confianza que proviene de la experiencia del consumidor.

Para Plummer (2000), la personalidad de la marca no es algo puramente físico o funcional, sino que las marcas pueden representar valores y adquirir características de personalidad humana. Pueden ser entonces descritas como jóvenes, cercanas, modernas, etc. Incluso auto- res como Sundar y Noseworthy (2016) establecen que esas características de la personalidad de la marca pueden afectar positivamente en diferentes aspectos del consumidor

Esta situación es relevante en el caso del deporte y, especialmente, en el fútbol, cuya esencia descansa en parte importante sobre sentimientos intangibles. Estas sensaciones a la vez se asocian a una serie de símbolos, nombres y personas que representan a un país o a un determinado equipo (Gladden et al., 1998). Además, la relación de los hinchas y sus equipos o selecciones favoritas se basa en la identidad y pertenencia (Boyle \& Magnusson, 2007), es decir, muchos de ellos se describen a sí mismos como pertenecientes a la comunidad debido a su participación en el grupo (Demiray \& Burnaz, 2019). Esto significa ser parte de una masa que busca los mismos objetivos y resultados, pero también significa diferenciarse de un espectador (Groeppel-Klein et al., 2008), ya que convertirse en un hincha es tener conexiones más profundas con el equipo de preferencia que una persona cualquiera (Gray \& Wert-Gray, 2012; Zillmann \& Paulus, 1993).

Uno de los máximos exponentes en el ámbito de la personalidad de la marca es Aaker (1997), quien desarrolló un marco de referencia teórico en el cual determinó el número y naturaleza de las dimensiones de una marca que serían válidas para comparar, estudiar y analizar sus efectos de manera metódica y científica. En la actualidad existen más de 7.500 trabajos académicos que han utilizado esta metodología en diferentes productos y servicios en todo el mundo.

El instrumento de Aaker (1997) permite identificar cinco dimensiones (sinceridad, excitante, competencia, sofisticado y rudeza), las cuales agrupan una serie de rasgos (Figura 1) que posibilitan establecer la interacción entre la mar- 
Figura 1

Modelo de Personalidad de Marca

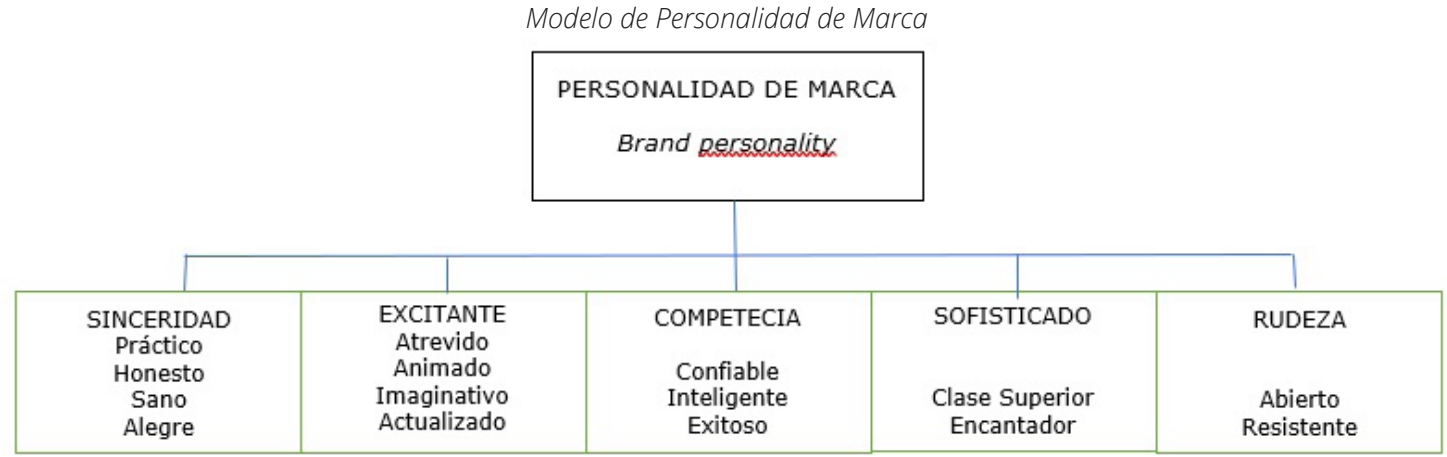

Nota: Aaker (1997)

ca y el consumidor mediante la atribución de rasgos de personalidad (Escobar \& Mateluna, 2016). Además, el modelo de Aaker (1997) resalta la importancia de construir marcas potentes basadas en la identidad y cómo esta puede desarrollarse.

Este listado de variables posibilita medir diferentes aspectos de manera sistemática, como las correlaciones entre la personalidad, la preferencia y el uso por parte de los consumidores. Estudiar las emociones que evoca en las personas una marca determinada y saber, por ejemplo, si aumentan o disminuyen los niveles de confianza y lealtad (Chernatony, 1997; Foumier, 1994).

De acuerdo a Aaker (1997) este aprendizaje contribuiría a una comprensión general del uso simbólico de las marcas, ya que proporcionaría una visión de las variables que influyen en la personalidad de la marca (Escobar \& Mateluna, 2016).

Un estudio realizado por Carlson et al. (2009) a las marcas en deportes, descubrió que las dimensiones asociadas a la sanidad y el éxito se asocian a la identificación que tienen las personas con su equipo, ya que una vez que el consumidor se reconoce como parte con un equipo, los gastos asociados (i.e. merchandising) tienden a aumentar. Mientras que Pan y
Phua (2020) confirmaron que el buen desempeño del equipo produjo efectos positivos en la confianza y la lealtad de la marca patrocinadora del club (Figura 1).

Lo mismo sucede con la transmisión de eventos que logran tener mayor audiencia. Algo que claramente se puede observar en Chile, dado que cuando juega la Selección Nacional de Fútbol, los canales de televisión logran obtener sus ratings más altos. Durante la última Copa Confederaciones disputada en Rusia 2017, las transmisiones bordearon los 60 puntos (Time Ibope) al igual que los últimos partidos eliminatorios para el Mundial 2018, al que Chile finalmente no clasificó.

Después de aplicar el marco conceptual de Aaker (1997), Braunstein y Ross (2010) Ilegaron a la conclusión de que conocer la personalidad de la marca permite a las organizaciones deportivas establecer estrategias diferenciadoras de los competidores $y$, de esa forma, destinar los recursos hacia la promoción en aquellas dimensiones que necesitan cambios o fortalecimiento de ciertos aspectos. Asimismo, permite conocer si los auspicios a los que se pretende asociar la organización deportiva tienen la conexión deseada.

Lee \& Cho (2009) exploraron qué marcas y eventos deportivos encajan mejor. Los resul- 
tados incluyeron a cerca de 400 personas y mostraron que el emparejamiento de marcas de "sinceridad" y "diligencia" produjo la mejor relación entre la marca, el evento y los consumidores. Mientras que Karjaluoto et al., (2016) establecieron que la identificación de los hinchas de hockey sobre hielo se relaciona con la lealtad y los años de relación. A menor cantidad de años, la personalidad de la marca se hace más relevante.

Kin et al., (2012) después de estudiar las marcas y el deporte concluyeron que un mejor entendimiento de la personalidad de marca permite a las organizaciones deportivas monitorear de manera más efectiva su efectividad y posicionamiento en el mercado, con el fin de conocer cómo evolucionan los consumidores y de esa manera moverse con ellos. Förster (2011) resalta la importancia de esa unión, porque es la única forma de crear una sólida identidad que permita ser reconocida por las personas, con el desafío de que los fanáticos de ese equipo o club se involucren activamente con la marca (Tsordia et al., 2018).

Couvelaere y Richelieu (2005) concluyen que el desarrollo e implementación de una estrategia de marca en el caso de los equipos de fútbol están correlacionados con los resultados deportivos que obtenga, pero que variables como la cercanía y el engagement son también relevantes. Por esa razón, se puede ver en los estadios de fútbol a miles de hinchas alentar a su equipo independientemente de que este vaya perdiendo o no esté en los primeros lugares de la tabla de posiciones.

\section{CHILE Y SU REALIDAD SOCIAL}

Con la apertura de la economía y desde el retorno de la democracia a principios de los noventa, Chile ha experimentado un notable desarrollo, con un PIB per cápita que se eleva como el más alto de América Latina, sobrepasando los US\$20 000 (Banco Mundial, 2019). Esto ha permitido una notable disminución de la pobreza, la cual alcanza en la actualidad al 10\% de los chilenos, siendo sólo Uruguay el país de la región que muestra mejores resultados en este ámbito (CEPAL, 2019).

En el plano institucional, y de acuerdo a Freedom House (2018), Chile no sólo se ubica como uno de los países de América Latina con mayor libertad política y civil, sino que también, y según Transparency Internacional (2019), como el segundo país de la región, tras Uruguay, con la menor percepción de la corrupción, posicionándolo entre las 30 naciones con mejores resultados a nivel mundial en este aspecto. Chile ingresó en 2010 en la Organización para la Cooperación y el Desarrollo Económico, (OCDE), considerada como el organismo que agrupa a los países desarrollados, siendo la primera nación sudamericana en hacerlo.

Estos logros en los planos económico e institucional deben ser contrastados con los altos niveles de desigualdad socioeconómica que aún persisten en la sociedad chilena (Banco Mundial, 2019). Como señala el PNUD (2017) sobre la desigualdad en Chile:

En Chile, decir que el país es desigual es una obviedad. La desigualdad es parte de su fisonomía histórica, un rasgo estructural del orden social desde sus inicios hasta nuestros días. Para los habitantes es un elemento esencial de cómo entienden el país donde viven y la posición que ocupan en la sociedad. (PNUD, 2017, p. 17)

Estas diferencias se traducen, por ejemplo, en que el $77 \%$ de los chilenos cree que la conflictividad entre ricos y pobres es fuerte o muy fuer- 
te; $77 \%$ señala no confiar en las demás personas (Latinobarómetro, 2017). Como explica el informe del Centro de Estudios Públicos (2016), esta desigualdad se ha traducido en un malestar constante de la población no solo con la institucionalidad del país, sino que también entre los chilenos.

\section{METODOLOGÍA}

Esta investigación tiene como propósito indagar qué percepciones tienen los chilenos respecto de su selección nacional y qué logra explicar el gran interés y conexión que tuvo el público inmediatamente después de que obtuviera las dos Copa América. Asimismo, se analizó el simbolismo de La Roja como elemento de unión de la sociedad chilena, a la que se considera como fragmentada.

A través de una encuesta diseñada y teniendo como base el modelo de personalidad de Aaker (1997), se buscó identificar y evaluar los atributos con que el público chileno reconoció a la selección nacional en su periodo de mayor éxito deportivo. Esta investigación se fundamenta en las siguientes preguntas:

Q1: La imagen de la selección chilena de fútbol es mejor que la imagen que se tiene del país

Q2: Los grupos socioeconómicos más bajos se sienten más representados por la selección chilena de fútbol

Q3: Los niños y jóvenes son más optimistas respecto de que la selección chilena de fútbol tendrá siempre mejores resultados

De esta manera, se encuestaron cara a cara a 2410 personas entre 10 y 80 años ubicadas en la Región Metropolitana y pertenecientes a diferentes segmentos socioeconómicos según
Tabla 1

Población por edad en la Región Metropolitana

\begin{tabular}{|c|c|c|c|}
\multicolumn{2}{c}{ Segmentos de etarios } & Hombre & Mujer \\
\hline Menores & $13-18$ & 287.968 & 278.790 \\
\hline Jóvenes & $19-25$ & 393.930 & 381.997 \\
\hline Adulto joven & $26-40$ & 854.643 & 858.526 \\
\hline Adulto & $41-65$ & 1.056 .156 & 970.897 \\
\hline Adulto mayor & $66-70$ & 111.436 & 305.517 \\
\hline & & 2.704 .133 & 2.795 .727 \\
\hline
\end{tabular}

Nota: Instituto Nacional de Estadísticas (2018)

Tabla 2

Segmentación por comuna

\begin{tabular}{|c|c|c|}
\multicolumn{2}{|c|}{$\begin{array}{c}\text { Comunas represen- } \\
\text { tativas del segmento } \\
\text { socioeconómico }\end{array}$} & $\begin{array}{c}\% \text { del to- } \\
\text { tal de la } \\
\text { población }\end{array}$ \\
\hline ABC1 & Prov - LC - Vitacura & $9 \%$ \\
\hline C2 & La Florida - Maipú - San Miguel & $20 \%$ \\
\hline & $\begin{array}{c}\text { San Bernardo - Est. Central - } \\
\text { Cerrillos }\end{array}$ & $33 \%$ \\
\hline C3 & Prov - Ñuñoa & $32 \%$ \\
\hline D & La Pintana & $6 \%$ \\
\hline E & & \\
\hline
\end{tabular}

Nota: AIM

distribución AIM. Este número fue ponderado de acuerdo al peso específico de cada segmento en la población (ver Tabla 1 y 2).

La participación en este estudio fue voluntaria y no fue necesario que las personas entregaran sus datos personales ni de contacto. Por lo tanto, era imposible después identificarlas ni individualizar sus respuestas. Tampoco se realizó pago alguno. La participación de los menores de edad fue en presencia de sus padres y/o un adulto.

El acceso a los datos sólo lo tuvieron los investigadores a cargo y los resultados se mantuvieron en un computador con clave al que no tenía acceso nadie que no fuera parte de este proyecto. 
Los encuestadores realizaron sus preguntas apoyándose en dispositivos móviles a los que se les cargó un software que permitía almacenar la información de manera directa en una nube de datos, a la que se podría acceder solamente con un nombre de usuario y clave determinada con el fin de proteger la confidencialidad de estos y para evitar errores posteriores en el proceso de tabulación.

A las personas que realizaron la encuesta se les realizó una capacitación en el manejo de los dispositivos y del software. Además, este programa contaba con un tutorial que podía ser consultado online.

Antes de comenzar con la encuesta se realizó un pilotaje de esta entre alumnos universitarios para corroborar la comprensión de las preguntas, que se cumplieran los objetivos y que el proceso técnico fuera el adecuado y funcionara correctamente.

El cuestionario basado en las dimensiones de Aaker (1997) y que se ha usado en distintas investigaciones en español (Martínez et al., 2005; Petroll et al., 2008), constó de 20 preguntas con escala Likert de 1 a 7. Esta escala facilita las respuestas en Chile, porque es la misma escala que se utiliza para las calificaciones escolares y de educación superior (universitaria y técnica).

\section{RESULTADOS}

Los resultados que se presentan a continuación deben ser comprendidos a partir de que existe una alta identificación de la muestra con la selección de Chile. Sobre el 70\% de todos los grupos socioeconómicos, y más del 80\% en el grupo D, señalaron sentirse representados con el equipo de fútbol (Tabla 3).

Esta misma representación también se produce según los rangos etarios. Desde los niños

\section{Tabla 3}

"Cuando juega la Selección, ¿te sientes repre sentado?". Respuestas afirmativas.

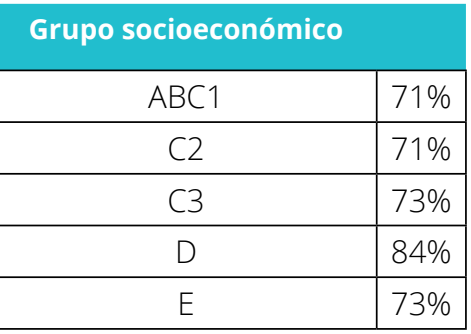

Tabla 4

"Cuando juega la Selección, ¿̇e sientes repre sentado?". Respuestas afirmativas.

\begin{tabular}{|c|c|}
\hline Grupo etario & $\%$ \\
\hline Adulto mayor & 76 \\
\hline Adulto & 75 \\
\hline Adulto joven & 78 \\
\hline Jóvenes & 78 \\
\hline Niños & 79 \\
\hline
\end{tabular}

hasta los adultos mayores mostraron una importante identificación con la Selección, en todos los casos en más de un 70\% (Tabla 4).

Es esta alta identificación, tanto de los grupos socioeconómicos como etarios, lo que permite establecer ciertas asociaciones entre el país y la Selección. Exitoso, eficiente, trabajador, auténtico, líder, fuerte y rudo fueron los principales rasgos, de los 15 que contempla el modelo de Aaker (1997), con los que el total de la muestra identificó a Chile y a La Roja (Tabla 5).

En general, se puede apreciar que la imagen del país y la Selección son similares, pero existe un reconocimiento de marca más fuerte en la Selección. Además, se puede establecer que, si bien las imágenes son similares, con respecto a la honestidad, se aprecia una diferencia de 24 puntos porcentuales. Ocurre algo similar con los adjetivos de: es exitoso, es líder y eficiente, con 28, 18 y 17 puntos de diferencia respecti- 
Tabla 5

Principales rasgos de personalidad de Aaker aplicados a Chile y su selección

\begin{tabular}{|c|c|c|c|c|c|}
\hline \multirow{4}{*}{$\begin{array}{c}\text { Personalidad } \\
\text { País }\end{array}$} & Exitoso & Eficiente & Trabajador & Trabajador & Fuerte \\
\cline { 2 - 6 } & Líder & Auténtico & Esforzado & Exitoso & Rudo \\
\hline \multirow{2}{*}{$\begin{array}{c}\text { Personalidad } \\
\text { Selección }\end{array}$} & Esforzado & Eficiente & Eficiente & Trabajador & Exitoso \\
\cline { 2 - 6 } & Líder & Líder & Líder & Líder & Líder \\
\hline
\end{tabular}

vamente entre la imagen de Chile y la selección chilena.

Con esta información se cumple la pregunta planteada respecto de que la imagen de la selección chilena de fútbol es mejor a la que se tiene del país. Esto ve reflejado, tal como se dijo anteriormente, en la masiva asistencia a los partidos de fútbol y en el alto rating de las transmisiones de los partidos. En contraste, la imagen del país se ha ido haciendo más inestable durante los últimos años. Desde 2013, se ha producido una importante baja sobre quie- nes creen que el país está progresando (UCGFK, 2018) (Figura 2).

Entre los distintos grupos socioeconómicos hubo consenso en caracterizar a la Selección como líder, rasgo que solo el grupo ABC1 consideró que también se aplicaba a Chile. Esto podría relacionarse con que este segmento identificó su situación socioeconómica con los éxitos deportivos del equipo nacional. Así también ocurre con la apreciación de los C2, que consideró eficientes tanto al país como al equipo.

Figura 2

Imagen país vs. imagen selección chilena de fútbol

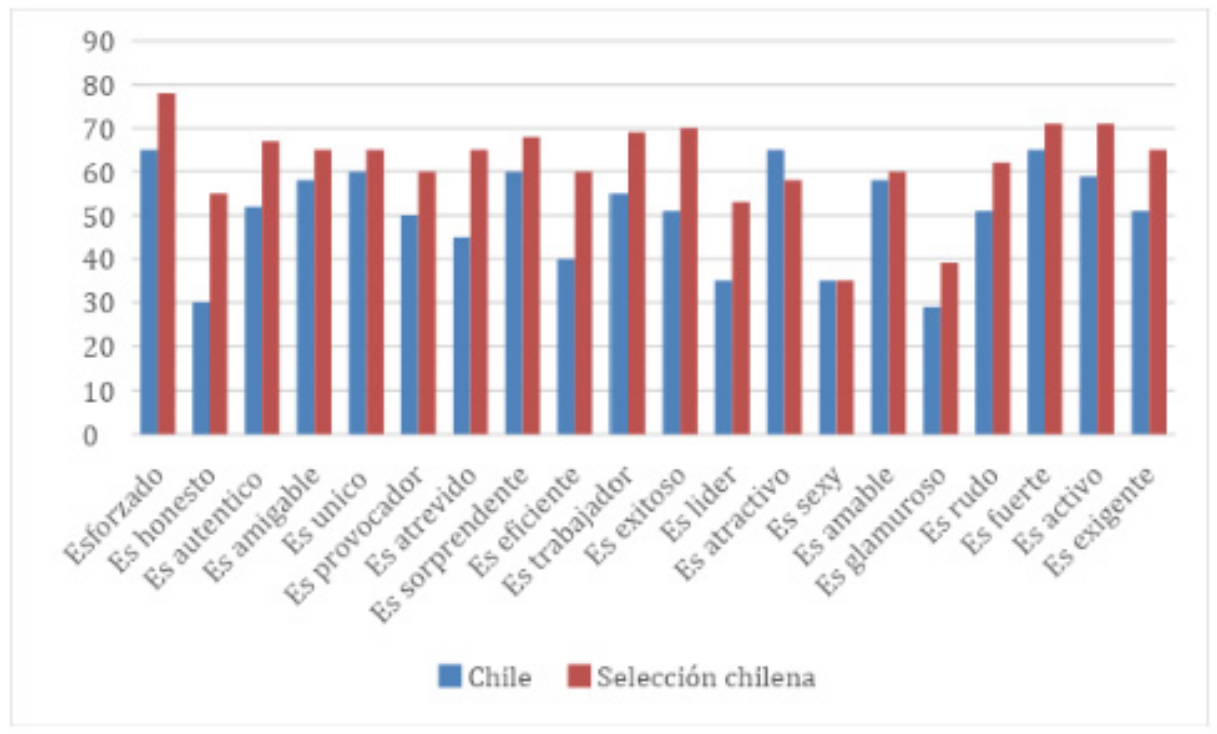


Por otra parte, se puede advertir que el grupo socioeconómico de menor ingreso utilizó características propias de un ambiente de adversidad para calificar al país, aunque mantuvo una apreciación de la Selección como exitosa. Diferente fue lo que ocurrió con el grupo D, que caracterizó tanto a Chile como al equipo como "trabajador".

Se realizó un test de Chi Cuadrado para cada par de variables de Chile y selección chilena, el resultado fue significativo en cada uno de los casos.

Otro rasgo de personalidad consultado fue con qué animal se identificaba a la selección de fútbol. Hubo acuerdo en que el perro representaba mejor las cualidades de La Roja. La elección de este animal puede tener una doble explicación. La primera, respecto al hecho de que a uno de los jugadores más importantes del equipo, y que posee una biografía de superación personal, se le apoda como El Pitbull. La segunda, y también plausible, es el simbolismo que tiene en el país el perro callejero, que debe arreglárselas diariamente para sobrevivir, al que se le llama comúnmente como quiltro, palabra que significa can en mapudungún. Otros animales que también destacaron fueron dos de los depredadores más feroces de la naturaleza, el león y el tigre.

Se puede establecer que estos rasgos de personalidad con los que se asocia a la selección nacional son reflejo de su rendimiento deportivo de los últimos años. Así, grupos como el ABC1 y el C3 creen que la Selección siempre va a ganar (Tabla 6).

Esto se debe a que la actual sociedad chilena es más optimista respecto a esta selección que a otras del pasado, sobre todo las nuevas generaciones. De acuerdo a la Tabla 6, son los niños de todos los grupos socioeconómicos los más optimistas con la actual selección, lo que

\section{Tabla 6}

"Cuando juega la Selección pensamos que ésta siem pre va a ganar. Respuestas afirmativas.

\begin{tabular}{|c|c|}
\hline Segmento & $\%$ \\
\hline$A B C 1$ & 56 \\
\hline$C 2$ & 38 \\
\hline$C 3$ & 52 \\
\hline$D$ & 38 \\
\hline$E$ & 48 \\
\hline
\end{tabular}

se puede explicar por el hecho de que no vivieron las derrotas de periodos anteriores (Tabla 7).

Este optimismo se traduce en que fueron los grupos que han vivido únicamente esta etapa ganadora de la Selección los más confiados en que el equipo llegaría a la final de la Copa del Mundo 2018 (Tabla 7). Los menores y los jóvenes, con un $31 \%$ y un $22 \%$ respectivamente, tuvieron más expectativas favorables sobre los resultados futuros del equipo, mientras que los adultos y los adultos mayores, con un 16\% y $19 \%$ respectivamente, presentaron menor confianza (Figura 3).

\section{Figura 3}

"Eres más optimista hoy que antes del rendimiento de la Selección nacional de fúbol?". Porcentaje de respuestas a firmativas según grupo socioeconómico y edad.

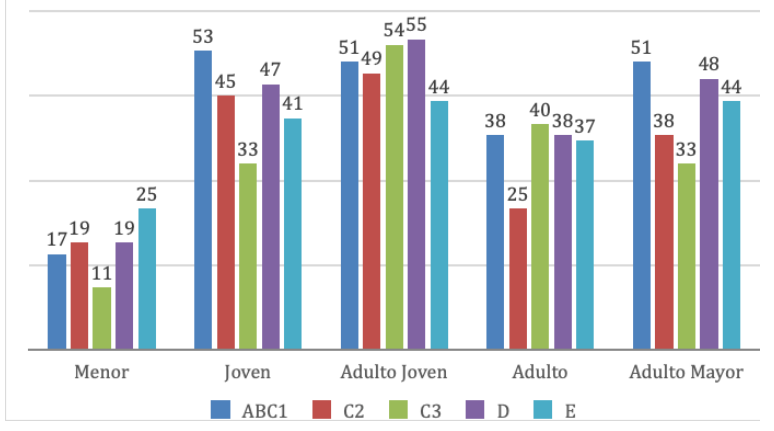

Respecto a la segunda pregunta de investigación, si los grupos socioeconómicos más bajos se sienten más representados por la selección chilena de fútbol, esta no se cumple. El grupo 
Tabla 7

Expectativas de la selección nacional para el mundial de fútbol.

\begin{tabular}{|c|c|c|c|c|c|c|}
\hline & No clasifica & $\begin{array}{c}\text { Primera } \\
\text { Ronda }\end{array}$ & $\begin{array}{l}\text { Octavos } \\
\text { de final }\end{array}$ & $\begin{array}{l}\text { Cuartos } \\
\text { de final }\end{array}$ & Semifinal & Final \\
\hline Adultos mayores & $25 \%$ & $29 \%$ & $24 \%$ & $20 \%$ & $20 \%$ & $19 \%$ \\
\hline Adulto & $19 \%$ & $24 \%$ & $18 \%$ & $21 \%$ & $16 \%$ & $16 \%$ \\
\hline Adulto joven & $10 \%$ & $10 \%$ & $9 \%$ & $13 \%$ & $15 \%$ & $12 \%$ \\
\hline Jóvenes & $22 \%$ & $22 \%$ & $23 \%$ & $27 \%$ & $25 \%$ & $22 \%$ \\
\hline Niños & $24 \%$ & $15 \%$ & $26 \%$ & $18 \%$ & $24 \%$ & $31 \%$ \\
\hline
\end{tabular}

socioeconómico más alto es el que se siente más representado por la Selección, seguido del grupo D y el que se siente menos representado es el grupo E y C3 (Figura 4). Ahora, en general, vemos que todos los grupos socioeconómicos afirman sentirse representados por la Selección. La misma tendencia ocurre sobre si creen que el estado de ánimo mejora cuando juega la Selección. Nuevamente el grupo ABC1 es el que se encuentra más de acuerdo con esta afirmación (Figura 4). Al realizar una prueba de Chi Cuadrado vemos que para ambas preguntas las diferencias son significativas estadísticamente a un 95\% de confianza $(p<0,05)$.

\section{Figura 4}

Representación y estado de ánimo por grupo socioeconómico

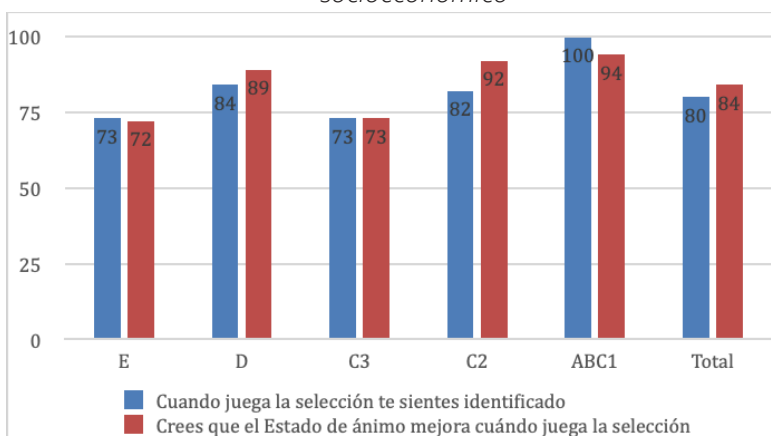

Al analizar la tercera pregunta de investigación, si los niños y jóvenes son más optimistas respecto de que la selección chilena de fútbol tendrá siempre mejores resultados. Esta se puede comprobar a través de los siguientes resultados.

Los menores y los jóvenes, con un 41\% y 45\% respectivamente, los que más consideraron que la selección campeona de la Copa América 2015 ha sido la mejor de la historia del fútbol chileno. Cabe destacar que los adultos mayores también estimaron como una de las mejores a la selección que ocupó el tercer lugar en la Copa Mundial de 1962, que se realizó en el país (Tabla 8).

Al preguntarles cuando juega la selección chilena, da lo mismo, el rival siempre va a ganar, encontramos que las expectativas de las personas son distintas a nivel estadístico dependiendo de la edad $(P<0,05, T=-2,019)$. Los más jóvenes poseen mayores expectativas sobre la Selección que las mayores. Al consultarles sobre el optimismo comparados con sus padres vemos que las diferencias no son estadísticamente significativas ( $P>0,05, T=-, 228)$, es decir, no existen diferencias estadísticamente significativas según la edad de las personas ante la frase "Tus padres eran más optimistas que tú" (Tabla 9). 
Tabla 8

"¿Cuál ha sido la mejor selección de fútbol de Chile?"

\begin{tabular}{|c|c|c|c|c|c|c|c|c|c|}
\hline & $\begin{array}{c}\text { Mundial } \\
1962\end{array}$ & $\begin{array}{c}\text { Mundial } \\
1974\end{array}$ & $\begin{array}{c}\text { Mundial } \\
1982\end{array}$ & $\begin{array}{c}\text { Copa } \\
\text { América } \\
1987\end{array}$ & $\begin{array}{c}\text { Mundial } \\
1998\end{array}$ & $\begin{array}{c}\text { Mundial } \\
2010\end{array}$ & $\begin{array}{c}\text { Mundial } \\
2014\end{array}$ & $\begin{array}{c}\text { Copa América } \\
2015\end{array}$ & $\begin{array}{c}\text { Copa } \\
\text { América } \\
2016\end{array}$ \\
\hline $\begin{array}{c}\text { Adultos } \\
\text { mayores }\end{array}$ & $27 \%$ & $2 \%$ & $4 \%$ & $2 \%$ & $7 \%$ & $9 \%$ & $8 \%$ & $29 \%$ & $12 \%$ \\
\hline Adultos & $10 \%$ & $4 \%$ & $6 \%$ & $1 \%$ & $4 \%$ & $8 \%$ & $11 \%$ & $36 \%$ & $23 \%$ \\
\hline $\begin{array}{c}\text { Adulto } \\
\text { Joven }\end{array}$ & $7 \%$ & $2 \%$ & $3 \%$ & $1 \%$ & $5 \%$ & $7 \%$ & $15 \%$ & $42 \%$ & $18 \%$ \\
\hline Jóvenes & $4 \%$ & $1 \%$ & $2 \%$ & $2 \%$ & $4 \%$ & $8 \%$ & $16 \%$ & $45 \%$ & $18 \%$ \\
\hline Niños & $4 \%$ & $2 \%$ & $1 \%$ & $1 \%$ & $3 \%$ & $13 \%$ & $14 \%$ & $41 \%$ & $21 \%$ \\
\hline
\end{tabular}

Tabla 9

"¿Cuál ha sido la mejor selección de fútbol de Chile?"

\begin{tabular}{ccccc} 
& & N & Media & $\begin{array}{c}\text { Desviación } \\
\text { estándar }\end{array}$ \\
\hline & Sí & 1036 & 34,8 & 20,3 \\
& No & 1374 & 36,5 & 20,4 \\
\hline
\end{tabular}

Tabla 10

"¿Tus padres eran más optimistas que tú?"

\begin{tabular}{ccccc} 
& & N & Media & $\begin{array}{c}\text { Desviación } \\
\text { estándar }\end{array}$ \\
Edad & Sí & 970 & 35,7 & 20,3 \\
& No & 1439 & 35,9 & 20,4 \\
\hline
\end{tabular}

Esta confianza deportiva respecto a la Selección puede llevarse a planos sociales, considerando que ella sirve de lazo de unión entre los diferentes grupos socioeconómicos y etarios. El 79\% de los consultados respondió que sí a la pregunta de si "la Selección nos une como país". No obstante, existió una diferencia considerable entre los grupos socioeconómicos más distantes. Mientras el ABC1, en todos los rangos etarios, consideró que la Selección nos une en más de un 90\%, en el grupo E — salvo en los niños, que exhiben un porcentaje similar al ABC1 - los porcentajes fueron menores, lle- gando en los jóvenes y adultos mayores a menos del 60\%.

\section{Figura 5}

"¿La selección de fútbol nos une como país?". Porcentaje de respuestas afirmativas.

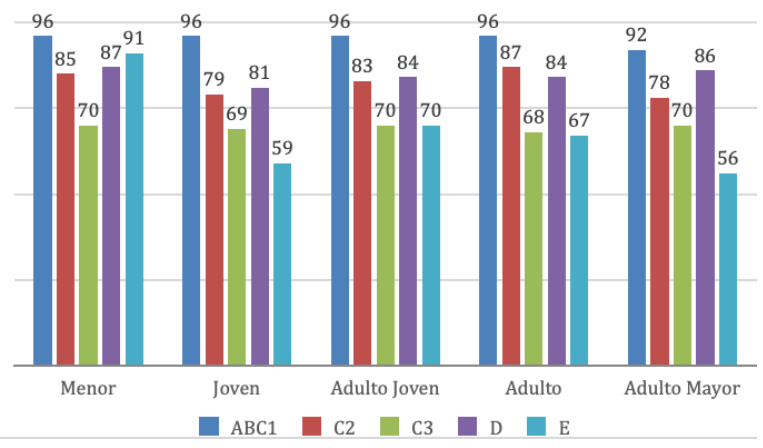

El 70\% de la muestra estimó que la Selección deja de lado las diferencias sociales. Al cruzar los grupos socioeconómicos con los rangos etarios, el resultado fue que el ABC1, en todas las edades, fue el segmento que más creyó que la Selección permite dejar de lado las diferencias sociales. Mientras que fueron los adultos del grupo D los que tuvieron una posición más negativa frente a esta pregunta. 
Figura 6

¿La selección de fútbol deja de lado las diferencias sociales?". Porcentaje de respuestas afirmativas.

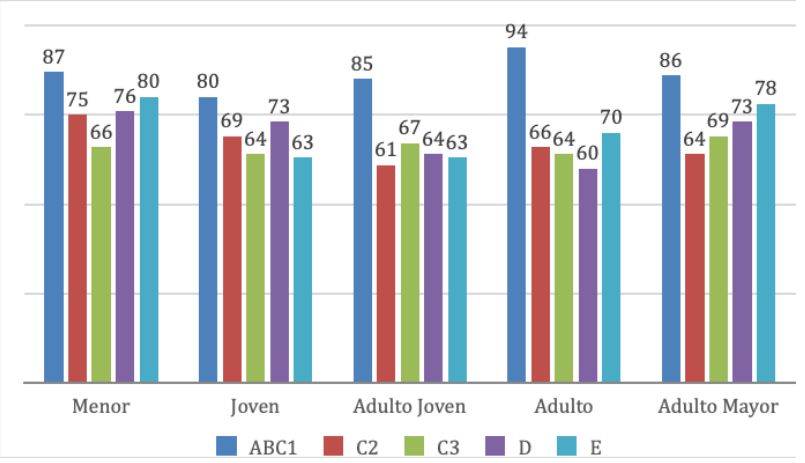

Es importante señalar que tanto en la pregunta de si la Selección une al país como en la que interroga si ella deja de lado las diferencias sociales, fue el grupo ABC1, en todos los rangos etarios, el que mostró una mayor tendencia positiva, mientras en los demás grupos, si bien hubo una importante aprobación a estos ítems, esta fue menor.

La importancia social que tiene la Selección lleva también a que aspectos simbólicos relacionados con la identidad del país sean asociados a ella. Generalmente, los equipos de las selecciones nacionales de fútbol cantan el himno patrio antes de comenzar el juego. Así, a la pregunta de si esto produce emoción, el 79\% de los encuestados declaró que sí. Sin embargo, en el cruce de los grupos socioeconómicos con el rango etario, el grupo E en todas las edades señaló una menor emocionalidad con este canto, llegando sólo al 50\% en los jóvenes. Entre las diferentes causas que podrían explicar este último dato, por ejemplo, podría estar el desapego de la juventud con la tradición, cuestión que no atañe propiamente a esta investigación.
Figura 7

¿La selección de fútbol deja de lado las diferencias sociales?". Porcentaje de respuestas afirmativas.

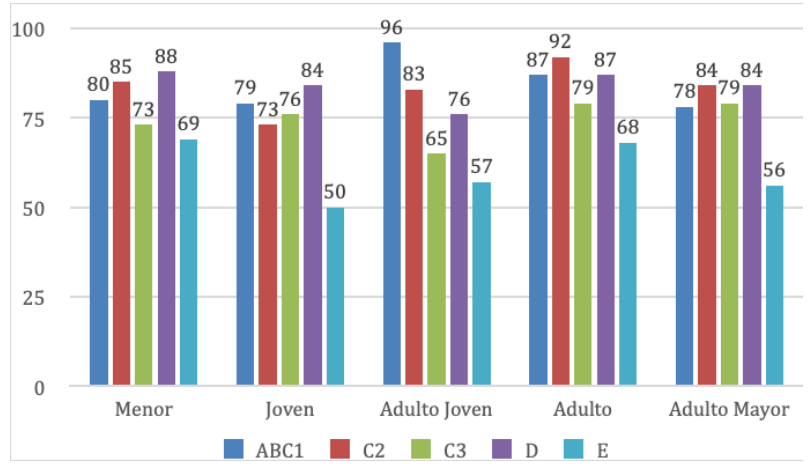

Respecto de una serie de actitudes, y según el total de la muestra, fue posible advertir que la selección de fútbol ha generado actitudes positivas hacia ella. Los chilenos se sienten más orgullosos de La Roja y la consideran más cercana a la gente que antes. Asimismo, a la Selección se le ponderó como más ganadora que las anteriores: el 80\% la consideró hoy más triunfadora, mientras que el 59\% señaló que siempre lo había sido. Esta visión optimista de la Selección se refleja en que el 68\% cree que alguna vez ella ganará la Copa Mundial de Fútbol. El aspecto negativo fue que un porcentaje alto, al igual que en el pasado, aún la considera como un negocio.

\section{Figura 8}

Actitudes respecto de la selección chilena

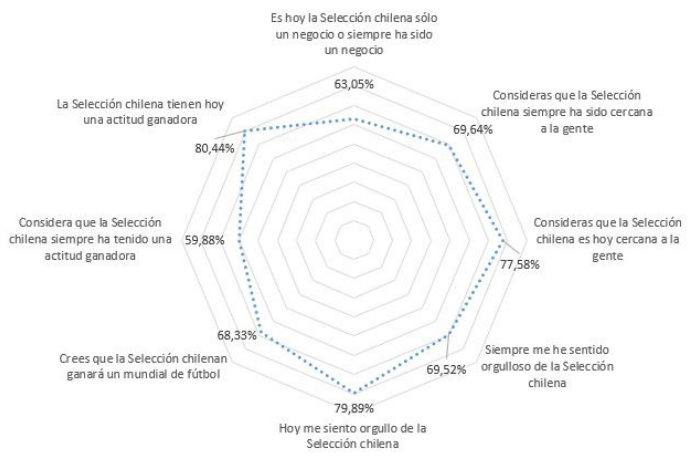


Al personalizar la Selección en sus jugadores, la apreciación del total de la muestra es positiva. Así, el 72\% los consideró como modelos para la juventud, el 82\% los estimó como ejemplos de superación y el $81 \%$ creyó que ellos juegan por el país. Por otra parte, si bien el 71\% consideró que juegan por dinero, un 78\% señala que son profesionales. En una proporción alta, pero menor, el 69\% los ve como ejemplos de vida.

\section{Figura 9}

Actitudes respecto de los jugadores de la selección chilena

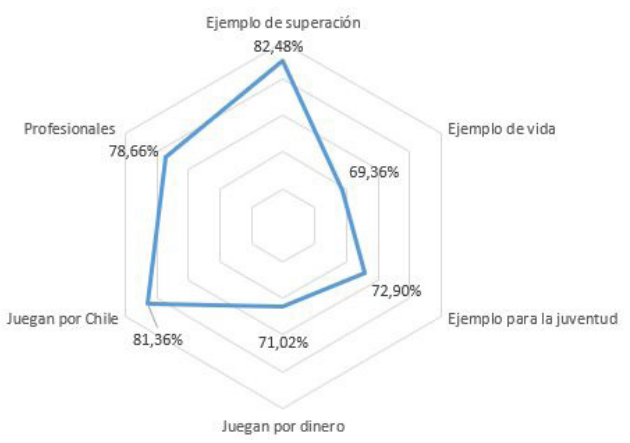

Al investigar las sensaciones que genera la selección chilena de fútbol se crearon 2 variables. La variable positiva es la suma de las variables: pasión, felicidad, placer, amor a la patria, confianza y admiración. La variable negativa es la suma de las variables: enojo, descontento, tristeza, miedo, vergüenza y nervios. Esto suma los valores de la escala de 1 a 5, es decir, si una persona está muy en desacuerdo con los 6 adjetivos, sumará 6 en la variable creada, por el contrario, si está muy de acuerdo con todos los adjetivos sumará 30. En los mínimos y máximos vemos que hay personas que estuvieron muy en desacuerdo con todos los adjetivos (sumaron 6) y otras que estuvieron muy de acuerdo con todos (sumaron 30). La media para los positivos es 22,3 y para los negativos 17,6 , esto quiere decir que las personas encuestadas afirman que la Selección les produce en mayor medida adjetivos positivos que negativos, pues mientras más cercana es la media a 30, quiere decir que más personas estuvieron de acuerdo con los adjetivos.

Tabla 11

Sensaciones que genera la selección chilena de fútbol

\begin{tabular}{cccccc} 
& N & Mínimo & Máximo & Media & $\begin{array}{c}\text { Desviación } \\
\text { estándar }\end{array}$ \\
$\begin{array}{c}\text { Posi- } \\
\text { tivo }\end{array}$ & 2331 & 6 & 30 & 22,3 & 4,8 \\
$\begin{array}{c}\text { Nega- } \\
\text { tivo }\end{array}$ & 2339 & 6 & 30 & 17,6 & 4,4 \\
\hline
\end{tabular}

En la tabla 12 se puede ver la correlación entre las variables creadas y la edad. Vemos que en ambos casos la correlación entre las variables es negativa, sin embargo, es muy débil al ser cercana a 0. Al observar la significancia vemos que es mayor a 0,05 por lo tanto fallamos en rechazar nuestra hipótesis nula y vemos que no hay relación lineal entre las variables positiva y negativa con la edad.

Tabla 12

Sensaciones por edad

\begin{tabular}{|c|c|c|c|c|}
\hline & & Edad & Positivo & Negativo \\
\hline Edad & $\begin{array}{l}\text { Correlación } \\
\text { de Pearson }\end{array}$ & 1 &,- 032 &,- 035 \\
\hline & $\begin{array}{c}\text { Sig. (bilate- } \\
\text { ral) }\end{array}$ & & , 122 & ,088 \\
\hline
\end{tabular}

\section{DISCUSIÓN}

Los resultados obtenidos en este trabajo revelan la fuerza como marca que tuvo la selección nacional más exitosa de todos los tiempos entre los chilenos. Si bien hay algunas diferencias entre grupos socioeconómicos y grupos etarios, en general la evaluación de La Roja y de las emociones que despierta es enormemente positiva, lo cual difiere de la imagen que la mayoría de los encuestados tiene del país. Así queda demostrado lo planteado por Blackket (2009), que establece que las marcas más potentes son aquellas que están construidas desde la base de la confianza y la experiencia. 
Tal como se mencionó, Chile fue el país que proporcionalmente llevó más público al estadio. Puede decirse que la alta demanda e interés por vivir la experiencia de ver a la Selección en vivo va de la mano con los resultados obtenidos en esta investigación.

El fervor que genera la selección de fútbol de Chile y los aspectos y sensaciones positivos demostrados en este trabajo podrían explicar por qué diferentes actores sociales y comerciales buscan asociarse con dicha marca. Un ejemplo de esto es el millonario contrato firmado el 2017 para adquirir sus derechos comerciales por el que se pagó un 20\% más que en el acuerdo anterior firmado cinco años antes (Contreras, 2017).

En lo particular, el estudio reveló que cada nivel socioeconómico identificó a la Selección y los valores que promueve desde su lugar de origen particular, haciendo de su positiva significación algo flexible que se corresponde con la realidad de cada grupo.

Respecto de los rangos etarios, fue posible observar que el optimismo de cada cohorte está relacionado con las vivencias, negativas o positivas, anteriores. Es por esa razón que los niños y jóvenes se muestran más optimistas, ya que han visto y vivido resultados casi únicamente favorables de la Selección. No sucede lo mismo con las generaciones mayores, que la vieron fracasar una y otra vez en diferentes competencias (clasificatorias, mundiales, Copa América, etc.).

Asimismo, se logró comprobar que la Selección une a los chilenos y es un elemento de cohesión social poco frecuente en nuestra convivencia actual. En este sentido, y teniendo en cuenta los altos índices de desigualdad y desconfianza en Chile, La Roja debe ser comprendida no sólo en lo que concierne a lo meramente deportivo, sino como una marca que promueve la buena convivencia y es lazo de unión entre las clases sociales y grupos etarios del país.

Estos atributos favorables se traspasan también a los jugadores seleccionados, ya que son percibidos como modelos a seguir. Se destacan los aspectos meritocráticos de sus vidas, siendo esta dimensión un aspecto relevante para la sociedad chilena, ya que la meritocracia se ha establecido como un aspecto relevante para la cohesión social (Siles, 2018).

\section{CONCLUSIONES Y APLICACIONES PRÁCTICAS}

A partir de las preguntas de investigación en las que se fundamentó esta investigación, se concluye:

1. Es posible establecer que la imagen del país y la selección son similares, pero existe un reconocimiento de marca más fuerte en el equipo. En este sentido, esto se puede explicar, por una parte, por los éxitos de La Roja entre los años 2014 a 2106, y por la otra, a las visiones negativas del país y su progreso de los últimos años.

2. Si bien la diferencia es pequeña, son los grupos socioeconómicos más altos los que se sienten más representados por la selección. Esto se podría explicar por una suerte de asimilación del éxito del equipo con el de sus propios logros económicos.

3. Los niños y los jóvenes son más optimistas con la selección y sus triunfos. Esto se debe a que las nuevas generaciones sólo han sido testigos de los logros de esta selección, no teniendo en la memoria, como sí ocurre con los grupos de mayor edad, las derrotas de los equipos pasados.

Sin duda, precisar las percepciones de la población chilena respecto del equipo más popular 
de fútbol del país permite establecer una serie de aplicaciones prácticas:

1. En cuanto a políticas públicas, el comprender las percepciones que genera La Roja en la sociedad abre la puerta para utilizarla para fomentar o desarrollar propuestas en deportes o vida sana, entre otros ámbitos. En este sentido, el estudio permite focalizar estas futuras políticas según rango etario y nivel socioeconómico, mejorando su focalización y precisión.

2. En cuanto a marca, los auspiciadores de la Selección tendrán acceso a un perfil más delimitado de las percepciones que genera el equipo, pudiendo orientar sus campañas publicitarias con el propósito de conseguir mayor impacto. Desde la perspectiva inversa, a la Selección en cuanto institución, el poseer conocimientos de su alcance en los grupos etarios y socioeconómicos le permite conocer su valorización como marca.

3. En cuanto a fenómeno social, para países con altos grados de desigualdad económica y desconfianza como Chile, contar con símbolos de unión como la selección de fútbol les permite generar a partir de ella políticas socioculturales orientadas a mejorar la convivencia y la confianza entre los ciudadanos.
En futuras investigaciones, y como parte de las limitaciones de ésta, podría ampliarse la muestra de participantes a regiones del país, de manera de constatar si los sentimientos que genera La Roja son similares en otras ciudades. Asimismo, se podría generar una comparación de si cambia la percepción cuando la selección es deportivamente exitosa a cuando ya deja de serlo. También se puede ampliar la muestra a equipos de fútbol locales, para saber si los atributos de esas marcas individuales son similares a los que posee el llamado "equipo de todos". 


\section{REFERENCIAS}

Aaker, J. L. (1997). Dimensions of brand personality. Journal of marketing research, 34(3), 347-356. https://doi.org/10.2307/3151897

Aaker, D. A., \& Biel, A. L. (1993). Brand equity and advertising: An overview (pp. 1-10). Lawrence Erlbaum Associates.

Acuña, G., \& Acuña, Á. (2016). El fútbol como producto cultural: Revisión y análisis bibliográfico. Citius, Altius, Fortius: Humanismo, Sociedad y Deporte: Investigaciones y ensayos (CAF). https://doi.org/10.15366/citius2016.9.2.003

Alabarces, P. (2003). Futbologías: fútbol, identidad y violencia en América Latina. Consejo Latinoamericano de Ciencias Sociales.

Allport, G. W. (1961). Pattern and growth in personality. Holt, Reinhart \& Winston.

Anderson, B. (1993). Comunidades imaginadas. Reflexiones sobre el origen y la difusión del nacionalismo [Imagined Communities: Reflections on the Origin and Spread of Nationalism]. Fondo de Cultura Económica.

Belair-Gagnon, V., Nelson, J. L., \& Lewis, S. C. (2019). Audience engagement, reciprocity, and the pursuit of community connectedness in public media journalism. Journalism Practice, 13(5), 558-575. https://doi.org/1 0.1080/17512786.2018.1542975

Blackett, T. (2009). What Makes Brand Great and Branding. London Profile Books.

Boyle, B. A., \& Magnusson, P. (2007). Social identity and brand equity formation: A comparative study of collegiate sports fans. Journal of Sport Management, 21(4), 497-520. https://doi.org/10.1123/jsm.21.4.497

Braunstein, J. R., \& Ross, S. D. (2010). Brand personality in sport: Dimension analysis and general scale development. Sport Marketing Quarterly, 19(1), 8-16.

Brodie, R. J., Ilic, A., Juric, B., \& Hollebeek, L. (2013). Consumer engagement in a virtual brand community: An exploratory analysis. Journal of business research, 66(1), 105-114. https://doi.org/10.1016/j.jbusres.2011.07.029

Carlson, B. D., Todd Donavan, D., \& Cumiskey, K. J. (2009). Consumer-brand relationships in sport: brand personality and identification. International Journal of Retail \& Distribution Management, 37(4), 370-384. https:// doi.org/10.1108/09590550910948592

Centro de Estudios Públicos (2016). Informe Encuesta CEP 2016. ¿Malestar en Chile? CEP. 
Comisión Económica para América Latina y el Caribe (CEPAL) (2019). Panorama Social de América Latina. CEPAL.

Contreras, G. (2017). Mediapro y Chilevisión cierran acuerdo por derechos comerciales de La Roja. La Tercera. https://www.latercera.com/el-deportivo/noticia/mediapro-chilevision-cierran-acuerdo-derechos-comerciales-la-roja/24144/

Couvelaere, V., \& Richelieu, A. (2005). Brand strategy in professional sports: The case of French soccer teams. European Sport Management Quartely, 5(1), 23-46. https://doi.org/10.1080/16184740500089524

Chernatony, L. D. (1997). Integrated brand building using brand taxonomies. Journal of Product and Brand Management, 6(1), 56-63. https://doi. org/10.1108/10610429710160048

Demiray, M., \& Burnaz, S. (2019). Exploring the impact of brand community identification on Facebook: firm-directed and self-directed drivers. Journal of Business Research, 96, 115-124. https://doi.org/10.1016/j. jbusres.2018.11.016

Doyle, P. (1990), "Building Successful Brands: The Strategic Options", Journal of Consumer Marketing, 7(2), 5-20. https://doi.org/10.1108/ EUM0000000002572

Escobar, M. \& Mateluna, C. (2016). Modelos de Personalidad de marca: una descripción de 1997 a 2015. Revista Academia \& Negocios, 2(2), 29-42.

Feller, Villanueva R. (2006). Planificación estratégica de marketing: Asociación Nacional de Fútbol Profesional ANFP 2007-2010. [Tesis Universidad de Chile].

Förster, K. (2011). Key success factors of TV brand management: An international case study analysis. Journal of media business studies, 8(4), 1-22. https://doi.org/10.1080/16522354.2011.11073528

Freedom House (2018). Freedom in the World 2018. Freedom House.

Gladden, J. M., Milne, G. R., \& Sutton, W. A. (1998). A conceptual framework for assessing brand equity in Division I college athletics. Journal of sport management, 12(1), 1-19. https://doi.org/10.1123/jsm.12.1.1

Gray, G. T., \& Wert-Gray, S. (2012). Customer retention in sports organization marketing: Examining the impact of team identification and satisfaction with team performance. International Journal of Consumer Studies, 36(3), 275-281. https://doi.org/10.1111/j.1470-6431.2011.00999.x

Groeppel-Klein, A., Koenigstorfer, J., \& Schmitt, M. (2008). You'll Never Walk Alone "-How Loyal Are Football Fans to Their Clubs When They Are Struggling against Relegation. Proceedings of the 16th EASM Conference, Bayreuth/Heidelberg. 
Hernández-Gil, C., Figueroa-Ramírez, E. F., \& Correa-Corrales, L. E. (2018). Reposicionamiento de marca: el camino hacia la competitividad de las pequeñas y medianas empresas. Revista de Investigación, Desarrollo e Innovación, 9(1), 33-46. https://doi.org/10.19053/20278306. v9.n1.2018.8505

Hite, K. (2004). Chile's National Stadium. As Monument, as Memorial. Revista. Harvard Review of Latin America, 3.

Karjaluoto, H., Munnukka, J., \& Salmi, M. (2016). How do brand personality, identification, and relationship length drive loyalty in sports? Journal of Service Theory and Practice, 26(1), 50-71. https://doi.org/10.1108/JSTP09-2014-0206

Kim, Y., Magnusen, M., \& Kim, Y. (2012). Revisiting sport brand personality: Scale development and validation. Journal of Multidisciplinary Research, $4(3), 65$.

Latinobarómetro (2017). Informe Latinobarómetro 2017. Latinobarómetro.

Lee, H. S., \& Cho, C. H. (2009). The matching effect of brand and sporting event personality: Sponsorship implications. Journal of Sport Management, 23(1), 41-64. https://doi.org/10.1123/jsm.23.1.41

Matamala, D. (2001). Goles y autogoles: la impropia relación entre el fútbol y el poder político. Planeta.

Martínez, E., Montaner, T., \& Piña, J. M. (2005). Propuesta de medición de la imagen de marca: un análisis aplicado a las extensiones de marca. Revista Asturiana de Economía, (33), 89-112.

Pan, P.-L. and Phua, J. (2020). Connecting sponsor brands through sports competitions: an identity approach to brand trust and brand loyalty. Sport, Business and Management, Vol. ahead-of-print No. ahead-of-print. https://doi.org/10.1108/SBM-01-2019-0003

Peña, C. (2020). Pensar el malestar: La crisis de octubre y la cuestión constitucional. TAURUS.

Petroll, M. D. L. M., Damacena, C., \& Merino, M. H. (2008). Medición y determinantes del valor de marca en la perspectiva del consumidor. Contabilidad y negocios, 3(6), 19-37.

Plummer, J. T. (2000). How personality makes a difference. Journal of advertising research, 40(6), 79-83. https://doi.org/10.2501/JAR-40-6-79-83

PNUD (2017). Desiguales. Orígenes, cambios y desafíos de la brecha social en Chile. PNUD.

Rather, R.A., Tehseen, S. and Parrey, S.H. (2018), "Promoting customer brand engagement and brand loyalty through customer brand identification and value congruity", Spanish Journal of Marketing - ESIC, 22(3), 319-337. https://doi.org/10.1108/SJME-06-2018-0030 
Santa Cruz, E. (2010). "De sentimiento popular a marca registrada. Fútbol, identidad y nacionalismo en Chile". En Cid, G., y San Francisco, A. (2010). Nacionalismos e identidad nacional en Chile: sig/o XX. Centro de Estudios Bicentenario.

Santos, T. C. (2003). O lado 'hard'da cultura 'cool': as torcidas e a violência no futebol. Alabarces, P. Futbologías: fútbol, identidad y violencia em América Latina. CLACSO.

Sertsios, G. (2007). ¿Puede el diseño de un torneo deportivo afectar su asistencia? Cuadernos de economía, 44(129), 59-89. http://dx.doi. org/10.4067/S0717-68212007000100003

Siles, C. (2018). Desigualdad, élites y encuentro. Algunos desafíos para la cohesión social en Chile. Estudios Públicos, 149, 75-103.

Sundar, Aparna, Noseworthy, Theodore J. (2016), Too Exciting to Fail, Too Sincere to Succeed: The Effects of Brand Personality on Sensory Disconfirmation, Journal of Consumer Research, 43(1), 44-67. https://doi. org/10.1093/jcr/ucw003

Tapia M.J. (2019). El fútbol chileno sale a la caza de las marcas que se cuelgan de su imagen. https://www.latercera.com/pulso/noticia/futbol-chilenosale-la-caza-las-marcas-se-cuelgan-imagen/757249/

Transparency International (2019). Corruption Perceptions Index 2018. Transparency International.

Tsordia, C., Papadimitriou, D., \& Parganas, P. (2018). The influence of sport sponsorship on brand equity and purchase behavior. Journal of Strategic Marketing, 26(1), 85-105. https://doi.org/10.1080/096525 4X.2017.1374299

UC y GfK Adimark, 2018. Encuesta Nacional Bicentenario (presentación). https://encuestabicentenario.uc.cl/wp-content/uploads/2018/10/Encuesta-bicentenario-2018-Sitio-Web.pdf

Villena, S. (2003). El fútbol y las identidades Prólogo a los estudios latinoamericanos. En CLACSO (2003) Futbologias: Futbol, identidad y violencia en America Latina. CLACSO.

World Bank (2019). World Bank national accounts data, and OECD National Accounts data files. World Bank.

Zillmann, D., \& Paulus, P. B. (1993). Spectators: Reactions to sports events and effects on athletic performance. Handbook of research on sport psychology, 600-619. 